\title{
Strong convergence of Halpern iterations for quasi-nonexpansive mappings and accretive operators in Banach spaces
}

\author{
Chin-Tzong Pang ${ }^{1}$ and Eskandar Naraghirad ${ }^{2 *}$
}

"Correspondence:
eskandarrad@gmail.com;
esnaraghirad@mail.yu.ac.ir
2Department of Mathematics,
Yasouj University, Yasouj, 75918, Iran
Full list of author information is
available at the end of the article

available at the end of the article

\begin{abstract}
In this paper, we first introduce a new Halpern-type iterative scheme to approximate common fixed points of an infinite family of quasi-nonexpansive mappings and obtain a strongly convergent iterative sequence to the common fixed points of these mappings in a uniformly convex Banach space. We then apply our method to approximate zeros of an infinite family of accretive operators and derive a strong convergence theorem for these operators. It is important to state clearly that the contribution of this paper in relation with the previous works (see, for example, Yao et al. (Nonlinear Anal. 70:2332-2336, 2009)) is a technical method to establish a strong convergence theorem of Halpern type for a wide class of quasi-nonexpansive mappings. The method provides a positive answer to an old problem in fixed point theory and applications. Our results improve and generalize many known results in the current literature.
\end{abstract}

MSC: $47 \mathrm{H} 10 ; 37 \mathrm{C} 25$

Keywords: accretive operator; fixed point; strong convergence; sunny nonexpansive retraction

\section{Introduction}

Throughout this paper, we denote the set of real numbers and the set of positive integers by $\mathbb{R}$ and $\mathbb{N}$, respectively. Let $E$ be a Banach space with the norm $\|\cdot\|$ and the dual space $E^{*}$. The modulus $\delta$ of convexity of $E$ is denoted by

$$
\delta(\epsilon)=\inf \left\{1-\frac{\|x+y\|}{2}:\|x\| \leq 1,\|y\| \leq 1,\|x-y\| \geq \epsilon\right\}
$$

for every $\epsilon$ with $0 \leq \epsilon \leq 2$. A Banach space $E$ is said to be uniformly convex if $\delta(\epsilon)>0$ for every $\epsilon>0$. Let $S_{E}=\{x \in E:\|x\|=1\}$. The norm of $E$ is said to be Gâteaux differentiable if for each $x, y \in S_{E}$, the limit

$$
\lim _{t \rightarrow 0} \frac{\|x+t y\|-\|x\|}{t}
$$

exists. In this case, $E$ is called smooth. If the limit (1.1) is attained uniformly in $x, y \in S_{E}$, then $E$ is called uniformly smooth. The Banach space $E$ is said to be strictly convex if $\left\|\frac{x+y}{2}\right\|<1$ whenever $x, y \in S_{E}$ and $x \neq y$. It is well known that $E$ is uniformly convex if and only if $E^{*}$ is

\section{空 Springer}

๑2013Pang and Naraghirad; licensee Springer. This is an Open Access article distributed under the terms of the Creative Commons Attribution License (http://creativecommons.org/licenses/by/2.0), which permits unrestricted use, distribution, and reproduction in any medium, provided the original work is properly cited. 
uniformly smooth. It is also known that if $E$ is reflexive, then $E$ is strictly convex if and only if $E^{*}$ is smooth; for more details, see [1]. Let $E$ be a Banach space with the norm $\|\cdot\|$ and the dual space $E^{*}$. When $\left\{x_{n}\right\}_{n \in \mathbb{N}}$ is a sequence in $E$, we denote the strong convergence of $\left\{x_{n}\right\}_{n \in \mathbb{N}}$ to $x \in E$ by $x_{n} \rightarrow x$ and the weak convergence by $x_{n} \rightarrow x$. For any sequence $\left\{x_{n}^{*}\right\}_{n \in \mathbb{N}}$ in $E^{*}$, we denote the strong convergence of $\left\{x_{n}^{*}\right\}_{n \in \mathbb{N}}$ to $x^{*} \in E^{*}$ by $x_{n}^{*} \rightarrow x^{*}$, the weak convergence by $x_{n}^{*} \rightarrow x^{*}$ and the weak-star convergence by $x_{n}^{*} \rightarrow^{*} x^{*}$. The normalized duality mapping $J: E \rightarrow 2^{E^{*}}$ is defined by

$$
J(x)=\left\{f \in E^{*}:\langle x, f\rangle=\|x\|^{2},\|x\|=\|f\|\right\}, \quad \forall x \in E .
$$

Now, we define a mapping $\rho:[0, \infty) \rightarrow[0, \infty)$, the modulus of smoothness of $E$, as follows:

$$
\rho(t)=\sup \left\{\frac{1}{2}(\|x+y\|+\|x-y\|)-1: x, y \in E,\|x\|=1,\|y\|=t\right\} .
$$

It is well known that $E$ is uniformly smooth if and only if $\lim _{t \rightarrow 0} \frac{\rho(t)}{t}=0$. Let $q \in \mathbb{R}$ be such that $1<q \leq 2$. Then a Banach space $E$ is said to be $q$-uniformly smooth if there exists a constant $c_{q}>0$ such that $\rho(t) \leq c_{q} t^{q}$ for all $t>0$. If a Banach space $E$ admits a sequentially continuous duality mapping $J$ from the weak topology to weak-star topology, then $J$ is single-valued and also $E$ is smooth; for more details, see [2,3]. In this case, the normalized duality mapping $J$ is said to be weakly sequentially continuous, i.e., if $\left\{x_{n}\right\}_{n \in \mathbb{N}} \subset E$ is a sequence with $x_{n} \rightarrow x \in E$, then $J\left(x_{n}\right) \rightarrow^{*} J(x)$ [2].

Let $E$ be a (real) Banach space and $C$ be a nonempty subset of $E$. Let $T: C \rightarrow E$ be a mapping. We denote by $F(T)$ the set of fixed points of $T$, i.e., $F(T)=\{x \in C: T x=x\}$. A mapping $T: C \rightarrow E$ is said to be nonexpansive if $\|T x-T y\| \leq\|x-y\|$ for all $x, y \in C$. A mapping $T: C \rightarrow E$ is said to be quasi-nonexpansive if $F(T) \neq \emptyset$ and $\|T x-y\| \leq\|x-y\|$ for all $x \in C$ and $y \in F(T)$.

In recent years, several types of iterative schemes have been constructed and proved in order to get strong convergence results for nonexpansive mappings in various settings. The concept of nonexpansivity plays an important role in the study of Halpern-type iteration for finding fixed points of a mapping $T: C \rightarrow C$. Recall that the one-step Halpern iteration is given by the following formula:

$$
x_{n+1}=\alpha_{n} u+\left(1-\alpha_{n}\right) T x_{n}, \quad u \in C, x_{1} \in C .
$$

Here, $\left\{\alpha_{n}\right\}_{n \in \mathbb{N}}$ is a real sequence in $[0,1]$ satisfying some appropriate conditions. A more general iteration scheme of one-step Halpern iteration is two-step Halpern iteration given by

$$
\left\{\begin{array}{l}
u \in C, \quad x_{1} \in C \quad \text { chosen arbitrarily, } \\
y_{n}=\left(1-\beta_{n}\right) x_{n}+\beta_{n} T x_{n} \\
x_{n+1}=\alpha_{n} u+\left(1-\alpha_{n}\right) y_{n}
\end{array}\right.
$$

where the sequences $\left\{\beta_{n}\right\}_{n \in \mathbb{N}}$ and $\left\{\alpha_{n}\right\}_{n \in \mathbb{N}}$ satisfy some appropriate conditions. In particular, when all $\beta_{n}=1$, the Halpern iteration (1.3) becomes the standard Halpern iteration (1.3). The construction of fixed points of nonexpansive mappings via Halpern's algorithm [4] has been extensively investigated recently in the current literature (see, for example, 
$[5,6]$ and the references therein). Because of a simple construction, Halpern's iterations are widely used to approximate a solution of fixed points for nonexpansive mappings and other classes of nonlinear mappings by many authors in different styles. In [7], Lions obtained a strong convergence result provided that the real sequence $\left\{\alpha_{n}\right\}_{n \in \mathbb{N}}$ satisfies the following conditions:

$$
\text { C1: } \quad \lim _{n \rightarrow \infty} \alpha_{n}=0 ; \quad \text { C2: } \quad \sum_{n=1}^{\infty} \alpha_{n}=\infty ; \quad \text { C3: } \quad \lim _{n \rightarrow \infty} \frac{\alpha_{n+1}-\alpha_{n}}{\alpha_{n+1}^{2}}=0 .
$$

In [5], Reich extended the result of Halpern from Hilbert spaces to uniformly smooth Banach spaces. However, both Halpern's and Lion's conditions imposed on the real sequence $\left\{\alpha_{n}\right\}_{n \in \mathbb{N}}$ exclude the canonical choice $\alpha_{n}=1 / n$ for all $n \in \mathbb{N}$. In [8], Wittmann proved strong convergence of $\left\{x_{n}\right\}_{n \in \mathbb{N}}$ if $\left\{\alpha_{n}\right\}_{n \in \mathbb{N}}$ satisfies the conditions $\mathrm{C} 1, \mathrm{C} 2$ and

$$
\text { C4: } \quad \sum_{n=1}^{\infty}\left|\alpha_{n+1}-\alpha_{n}\right|<\infty
$$

In [9], Shioji and Takahashi extended Wittmann's result from Hilbert spaces to real Banach spaces with uniformly Gâteaux differentiable norms and in which each nonempty, closed, convex and bounded subset has the fixed point property for nonexpansive mappings. In [10], Xu used another control condition C5: $\lim _{n \rightarrow \infty}\left(\alpha_{n+1}-\alpha_{n}\right) / \alpha_{n+1}=0$ instead of the conditions $\mathrm{C} 3$ or $\mathrm{C} 4$ and proved strong convergence of the sequence $\left\{x_{n}\right\}_{n \in \mathbb{N}}$. In [11], Cho, Kang and Zhou pointed out that the control conditions $\mathrm{C} 4$ and $\mathrm{C} 5$ are not comparable, in general. They proposed some examples which satisfy the conditions C1, C2, C3, C4 and C5, and also presented the control condition C6:

$$
\left|\alpha_{n+1}-\alpha_{n}\right| \leq o\left(\alpha_{n+1}\right)+\sigma_{n}
$$

where $\sum_{n=1}^{\infty} \sigma_{n}<\infty$. Recently, Suzuki [12] introduced the following Halpern-type iteration:

$$
\left\{\begin{array}{l}
u \in C, \quad x_{1} \in C \quad \text { chosen arbitrarily, } \\
y_{n}=\lambda x_{n}+(1-\lambda) T x_{n} \\
x_{n+1}=\alpha_{n} u+\left(1-\alpha_{n}\right) y_{n}
\end{array}\right.
$$

He obtained strong convergence results for the sequence $\left\{x_{n}\right\}_{n \in \mathbb{N}}$ generated by algorithm (1.4) when only the conditions $\mathrm{C} 1$ and $\mathrm{C} 2$ are imposed on the sequence $\left\{x_{n}\right\}_{n \in \mathbb{N}}$, see also [12]. Numerous results have been proved on Halpern's iterations for nonexpansive mappings in Hilbert and Banach spaces (see, e.g., [13-15]). However, the following question in its full statements remains unsolved.

Open question 1.1 Are the conditions $\mathrm{C} 1$ and $\mathrm{C} 2$ sufficient for the strong convergence of the sequence $\left\{x_{n}\right\}_{n \in \mathbb{N}}$ generated by the algorithm (1.2) for all quasi-nonexpansive mappings $T: C \rightarrow C$, where $C$ is a nonempty, closed and convex subset of a Banach space $E$ ?

The answer to this question is known to be negative for nonexpansive mappings in a Hilbert space setting [16]. 
Let $E$ be a real Banach space, and let $A: E \rightarrow 2^{E}$ be a mapping. The effective domain of $A$ is denoted by $\operatorname{dom}(A)$, that is, $\operatorname{dom}(A)=\{x \in E: A x \neq \emptyset\}$. The range of $A$ is denoted by $R(A)$. A multi-valued mapping $A$ is said to be accretive if for all $x, y \in E$, there exists $j \in J(x-y)$ such that $\langle x-y, j\rangle \geq 0$, where $J: E \rightarrow 2^{E^{*}}$ is the duality mapping. An accretive operator $A$ is $m$-accretive if $R(I+r A)=E$ for each $r \geq 0$. Throughout this paper, we assume that $A: E \rightarrow 2^{E}$ is an accretive operator and has a zero. For an accretive operator $A$ on $E$ and $r>0$, we may define a single-valued operator $J_{r}=(I+r A)^{-1}: E \rightarrow \operatorname{dom}(A)$, which is called the resolvent of $A$ for $r>0$. Assume $A^{-1} 0=\{x \in E: 0 \in A x\}$. It is known that $A^{-1} 0=F\left(J_{r}\right)$ for all $r>0$ (for more details, see, for example, $[1,17]$ ). The accretive operator has a close connection with equations of evolution. Typical examples can be found in the heat, wave, or Shrödinger equations. For some recent articles on the approximation of common zeros of accretive operators, we refer the readers to [6, 18-21].

In this paper, we first introduce a new Halpern-type iterative scheme to approximate common fixed points of an infinite family of quasi-nonexpansive mappings and obtain a strongly convergent iterative sequence to the common fixed points of these mappings in a uniformly convex Banach space. We then apply our method to approximate zeros of an infinite family of accretive operators and derive a strong convergence theorem for these operators. It is important to state clearly that the contribution of this paper in relation with the previous works (see, for example, [17]) is a technical method to establish a strong convergence theorem of Halpern type for a wide class of quasi-nonexpansive mappings. Consequently, Open question 1.1 is answered in the affirmative by modifying (1.2) in a Banach space setting. Our results improve and generalize many known results in the current literature; see, for example, [14, 17, 22, 23].

\section{Preliminaries}

In this section, we collect some lemmas which will be used in the proofs for the main results in the next section.

Let $C$ be a nonempty, closed and convex subset of a reflexive, strictly convex and smooth Banach space $E$. Then, for any $x \in E$, there exists a unique point $y_{0} \in C$ such that

$$
\left\|y_{0}-x\right\| \leq \min _{y \in C}\|y-x\|
$$

For more details, we refer the reader to [1]. The mapping $P_{C}: E \rightarrow C$ defined by $P_{C} x=y_{0}$ is called the metric projection from $E$ onto $C$. Let $x \in E$ and $z \in C$. Then it is well known that $z=P_{C} x$ if and only if

$$
\langle z-y, J(x-z)| \geq 0
$$

for all $y \in C$, where $J$ is the normalized duality mapping of $E$; see [1] for more details. It is also well known that if $P_{C}$ is a metric projection from a real Hilbert space $H$ onto a nonempty, closed and convex subset $C$, then $P_{C}$ is nonexpansive, but, in a general Banach space, this fact is not true; see [24] for more details.

Let $C$ and $D$ be nonempty subsets of a real Banach space $E$ with $D \subset C$. A mapping $Q_{D}: C \rightarrow D$ is said to be sunny if

$$
Q_{D}\left(Q_{D} x+t\left(x-Q_{D} x\right)\right)=Q_{D} x
$$


for each $x \in E$ and $t \geq 0$. A mapping $Q_{D}: C \rightarrow D$ is said to be a retraction if $Q_{D} x=x$ for each $x \in C$. For more details, see [25].

A Banach space $E$ is said to have a weakly continuous duality mapping [26] if there exists a gauge function $\phi$ such that the duality mapping $J_{\phi}$ is single-valued and weak-to-weak* sequentially continuous, where the gauge function $\phi:[0, \infty) \rightarrow[0, \infty)$ is a continuous strictly increasing function with $\phi(0)=0$ and $\lim _{t \rightarrow \infty} \phi(t)=\infty$ and the duality mapping $J_{\phi}$ is defined by

$$
J_{\phi}(x)=\left\{f \in E^{*}:\langle x, f\rangle=\|x\|\|f\|, \phi(\|x\|)=\|f\|\right\}, \quad \forall x \in E .
$$

Let

$$
\Phi(t)=\int_{0}^{t} \phi(\tau) d \tau, \quad t \geq 0
$$

Then $\Phi(k t) \leq k \phi(t)$ for all $0<k<1$.

Lemma 2.1 [10] Let E be a real Banach space having a weakly sequentially continuous duality mapping $J_{\phi}$ with a gauge function $\phi$. Then

$$
\Phi(\|x+y\|) \leq \Phi(\|x\|)+2\left\langle y, J_{\phi}(x+y)\right\rangle
$$

for all $x, y \in E$. In particular, if $J_{\phi}=J$, the normalized duality mapping of $E$, then

$$
\|x+y\|^{2} \leq\|x\|^{2}+2\langle y, J(x+y)\rangle
$$

for all $x, y \in E$.

Lemma 2.2 [2] Let E be a Banach space with a weakly sequentially continuous duality mapping $J_{\phi}$ with a gauge function $\phi$. Let $C$ be a nonempty, closed and convex subset of $E$, and let $T: C \rightarrow C$ be a nonexpansive mapping with $F(T) \neq \emptyset$. Then, for each $u \in C$, there exists $x \in F(T)$ such that

$$
\left\langle u-x, J_{\phi}(y-x)\right\rangle \leq 0, \quad \forall y \in F(T) .
$$

Lemma $2.3[10,27]$ Let E be a reflexive Banach space and have a weakly continuous duality map $J_{\phi}$ with a gauge function $\phi$. Let $C$ be a nonempty, closed and convex subset of $E$, and let $T: C \rightarrow C$ be a nonexpansive mapping. Fix $u \in C$ and $t \in(0,1)$. Let $x_{t} \in C$ be the unique solution in $C$ to the equation $x_{t}=t u+(1-t) T x_{t}$. Then $T$ has a fixed point if and only if $\left\{x_{t}\right\}_{t \in(0,1)}$ remains bounded as $t \rightarrow 0^{+}$, and in this case, $\left\{x_{t}\right\}_{t \in(0,1)}$ converges as $t \rightarrow 0^{+}$ strongly to a fixed point of $T$. If we define $Q: C \rightarrow F(T)$ by

$$
Q(u):=\lim _{t \rightarrow 0} x_{t}, \quad u \in C,
$$

then $Q(u)$ solves the variational inequality

$$
\left\langle u-Q(u), J_{\phi}(y-Q(u))\right\rangle \leq 0, \quad u \in C, y \in F(T),
$$

where $Q$ is the sunny nonexpansive retraction from $C$ onto $F(T)$. 
Definition 2.1 Let $C$ be a nonempty subset of a Banach space $E$ and $T: C \rightarrow E$ be a mapping. Then the mapping $T$ is said to be demiclosed at zero if, for any sequence $\left\{x_{n}\right\}_{n \in \mathbb{N}}$ in $C$ which converges weakly to $z$, and if $\left\|T x_{n}-x_{n}\right\| \rightarrow 0$ as $n \rightarrow \infty$, then $T z=z$.

Lemma 2.4 (Demiclosedness principle [28]) Let E be a reflexive Banach space, $C$ be a nonempty, closed and convex subset of $E$ and $T: C \rightarrow E$ be a nonexpansive mapping. Suppose that $E$ admits a weakly sequentially continuous duality mapping $J_{\phi}$ with a gauge function $\phi$. Then the mapping $I-T$ is demiclosed on $C$, where I is the identity mapping, i.e., if $\left\{x_{n}\right\}_{n \in \mathbb{N}}$ is a sequence in $C$ that converges weakly to $x$ and if $\left\{(I-T) x_{n}\right\}_{n \in \mathbb{N}}$ converges strongly to $y$, then $(I-T) x=y$; in particular, if $y=0$, then $x \in F(T)$.

Lemma 2.5 [1] Let E be a real Banach space, and let $A$ be an $m$-accretive operator on $E$. For $r>0$, let $J_{r}$ be the resolvent operator associated with $A$ and $r$. Then

$$
\left\|J_{\rho} x-J_{\sigma} x\right\| \leq \frac{|\rho-\sigma|}{\rho}\left\|x-J_{\rho} x\right\|
$$

for all $\rho, \sigma>0$ and $x \in E$.

Lemma 2.6 [29] Let $\left\{s_{n}\right\}_{n \in \mathbb{N}}$ be a sequence of nonnegative real numbers satisfying the inequality

$$
s_{n+1} \leq\left(1-\gamma_{n}\right) s_{n}+\gamma_{n} \delta_{n}, \quad \forall n \geq 0,
$$

where $\left\{\gamma_{n}\right\}_{n \in \mathbb{N}}$ and $\left\{\delta_{n}\right\}_{n \in \mathbb{N}}$ satisfy the conditions:

(i) $\left\{\gamma_{n}\right\}_{n \in \mathbb{N}} \subset[0,1]$ and $\sum_{n=1}^{\infty} \gamma_{n}=\infty$, or equivalently, $\prod_{n=1}^{\infty}\left(1-\gamma_{n}\right)=0$;

(ii) $\lim \sup _{n \rightarrow \infty} \delta_{n} \leq 0$, or

(ii)' $\sum_{n=1}^{\infty} \gamma_{n} \delta_{n}<\infty$. Then $\lim _{n \rightarrow \infty} s_{n}=0$.

Lemma 2.7 [30] Let $\left\{a_{n}\right\}_{n \in \mathbb{N}}$ be a sequence of real numbers such that there exists a subsequence $\left\{n_{i}\right\}_{i \in \mathbb{N}}$ of $\{n\}_{n \in \mathbb{N}}$ such that $a_{n_{i}}<a_{n_{i}+1}$ for all $i \in \mathbb{N}$. Then there exists a subsequence $\left\{m_{k}\right\}_{k \in \mathbb{N}} \subset \mathbb{N}$ such that $m_{k} \rightarrow \infty$ and the following properties are satisfied by all (sufficiently large) numbers $k \in \mathbb{N}$ :

$$
a_{m_{k}} \leq a_{m_{k}+1} \text { and } a_{k} \leq a_{m_{k}+1} \text {. }
$$

In fact, $m_{k}=\max \left\{j \leq k: a_{j}<a_{j+1}\right\}$.

Lemma 2.8 [31] Let E be a uniformly convex Banach space, $r>0$ be a constant. Then there exists a continuous, strictly increasing and convex function $g:[0,2 r) \rightarrow[0, \infty)$ such that

$$
\left\|\sum_{k=0}^{\infty} \alpha_{k} x_{k}\right\|^{2} \leq \sum_{k=0}^{\infty} \alpha_{k}\left\|x_{k}\right\|^{2}-\alpha_{i} \alpha_{j} g\left(\left\|x_{i}-x_{j}\right\|\right)
$$

for all $i, j \in \mathbb{N} \cup\{0\}, x_{k} \in B_{r}:=\{z \in E:\|z\| \leq r\}, \alpha_{k} \in(0,1)$ and $k \in \mathbb{N} \cup\{0\}$ with $\sum_{k=0}^{\infty} \alpha_{k}=1$. 


\section{Strong convergence theorems for quasi-nonexpansive mappings}

In this section, we prove a strong convergence theorem of Halpern type to approximate common fixed points of an infinite family of quasi-nonexpansive mappings in a uniformly convex Banach space.

Theorem 3.1 Let $E$ be a real uniformly convex Banach space with the normalized duality mapping J. Let $C$ be a nonempty, closed and convex subset of $E$, and let $\left\{T_{j}\right\}_{j \in \mathbb{N}}$ be an infinite family of quasi-nonexpansive mappings from $C$ into itself such that $F:=\bigcap_{j=1}^{\infty} F\left(T_{j}\right) \neq \emptyset$. Assume that for each $j \in \mathbb{N}, T_{j}-I$ is demiclosed at 0 . Let $\left\{\alpha_{n}\right\}_{n \in \mathbb{N}},\left\{\beta_{n, j}\right\}_{n \in \mathbb{N}, j \in \mathbb{N} \cup\{0\}}$ be sequences in $[0,1]$ satisfying the following control conditions:

(a) $\lim _{n \rightarrow \infty} \alpha_{n}=0$;

(b) $\sum_{n=1}^{\infty} \alpha_{n}=\infty$;

(c) $\beta_{n, 0}+\sum_{j=1}^{\infty} \beta_{n, j}=1, \forall n \in \mathbb{N}$;

(d) $\liminf _{n \rightarrow \infty} \beta_{n, 0} \beta_{n, j}>0, \forall j \in \mathbb{N}$.

Let $\left\{x_{n}\right\}_{n \in \mathbb{N}}$ be a sequence generated by

$$
\left\{\begin{array}{l}
x_{1}, u \in C \quad \text { chosen arbitrarily } \\
y_{n}=\beta_{n, 0} x_{n}+\sum_{j=1}^{\infty} \beta_{n, j} T_{j} x_{n} \\
x_{n+1}=\alpha_{n} u+\left(1-\alpha_{n}\right) y_{n} .
\end{array}\right.
$$

Then the sequence $\left\{x_{n}\right\}_{n \in \mathbb{N}}$ defined in (3.1) converges strongly to $P_{F} u$, where $P_{F}$ is the metric projection from $E$ onto $F$.

Proof We divide the proof into several steps.

We first note that $F$ is closed and convex. Set $z=P_{F} u$.

Step 1. We prove that the sequences $\left\{x_{n}\right\}_{n \in \mathbb{N}},\left\{y_{n}\right\}_{n \in \mathbb{N}}$ and $\left\{T_{j} x_{n}\right\}_{n \in \mathbb{N}}$ are bounded.

We first show that $\left\{x_{n}\right\}_{n \in \mathbb{N}}$ is bounded. Let $p \in F$ be fixed. In view of Lemma 2.8, there exists a continuous, strictly increasing and convex function $g:[0, \infty) \rightarrow[0, \infty)$ with $g(0)=$ 0 such that for any $j \in \mathbb{N}$,

$$
\begin{aligned}
\left\|y_{n}-p\right\|^{2} & =\left\|\beta_{n, 0} x_{n}+\sum_{j=1}^{\infty} \beta_{n, j} T_{j} x_{n}-p\right\|^{2} \\
& \leq \beta_{n, 0}\left\|x_{n}-p\right\|^{2}+\sum_{j=1}^{\infty} \beta_{n, j}\left\|T_{j} x_{n}-p\right\|^{2}-\beta_{n, 0} \beta_{n, j} g\left(\left\|x_{n}-T_{j} x_{n}\right\|\right) \\
& \leq \beta_{n, 0}\left\|x_{n}-p\right\|^{2}+\sum_{j=1}^{\infty} \beta_{n, j}\left\|x_{n}-p\right\|^{2}-\beta_{n, 0} \beta_{n, j} g\left(\left\|x_{n}-T_{j} x_{n}\right\|\right) \\
& =\left\|x_{n}-p\right\|^{2}-\beta_{n, 0} \beta_{n, j} g\left(\left\|x_{n}-T_{j} x_{n}\right\|\right) \\
& \leq\left\|x_{n}-p\right\|^{2} .
\end{aligned}
$$

This implies that

$$
\begin{aligned}
\left\|x_{n+1}-p\right\| & =\left\|\alpha_{n} u+\left(1-\alpha_{n}\right) y_{n}-p\right\| \leq \alpha_{n}\|u-p\|+\left(1-\alpha_{n}\right)\left\|y_{n}-p\right\| \\
& \leq \alpha_{n}\|u-p\|+\left(1-\alpha_{n}\right)\left\|x_{n}-p\right\| \leq \max \left\{\|u-p\|,\left\|x_{n}-p\right\|\right\} .
\end{aligned}
$$


By induction, we obtain

$$
\left\|x_{n+1}-p\right\| \leq \max \left\{\|u-p\|,\left\|x_{1}-p\right\|\right\}
$$

for all $n \in \mathbb{N}$. This implies that the sequence $\left\{\left\|x_{n}-p\right\|\right\}_{n \in \mathbb{N}}$ is bounded and hence the sequence $\left\{x_{n}\right\}_{n \in \mathbb{N}}$ is bounded. This, together with (3.1), implies that the sequences $\left\{y_{n}\right\}_{n \in \mathbb{N}}$ and $\left\{T_{j} x_{n}\right\}_{n \in \mathbb{N}}$ are bounded too.

Step 2. We prove that for any $n \in \mathbb{N}$,

$$
\left\|x_{n+1}-z\right\|^{2} \leq\left(1-\alpha_{n}\right)\left\|x_{n}-z\right\|^{2}+2 \alpha_{n}\left\langle u-z, J\left(x_{n+1}-z\right)\right\rangle .
$$

Let us show (3.3). For any $n, j \in \mathbb{N}$, in view of (3.2), we obtain

$$
\left\|y_{n}-z\right\|^{2} \leq\left\|x_{n}-z\right\|^{2}-\beta_{n, 0} \beta_{n, j} g\left(\left\|x_{n}-T_{j} x_{n}\right\|\right) .
$$

This implies that

$$
\begin{aligned}
\left\|x_{n+1}-z\right\|^{2} & =\left\|\alpha_{n} u+\left(1-\alpha_{n}\right) y_{n}-z\right\|^{2} \leq \alpha_{n}\|u-z\|^{2}+\left(1-\alpha_{n}\right)\left\|y_{n}-z\right\|^{2} \\
& \leq \alpha_{n}\|u-z\|^{2}+\left(1-\alpha_{n}\right)\left[\left\|x_{n}-z\right\|^{2}-\beta_{n, 0} \beta_{n, j} g\left(\left\|x_{n}-T_{j} x_{n}\right\|\right)\right] .
\end{aligned}
$$

Let $M_{1}:=\sup \left\{\left|\|u-z\|^{2}-\left\|x_{n}-z\right\|^{2}\right|+\beta_{n, 0} \beta_{n, j} g\left(\left\|x_{n}-T_{j} x_{n}\right\|\right): n, j \in \mathbb{N}\right\}$. It follows from (3.4) that

$$
\beta_{n, 0} \beta_{n, j} g\left(\left\|x_{n}-T_{j} x_{n}\right\|\right) \leq\left\|x_{n}-z\right\|^{2}-\left\|x_{n+1}-z\right\|^{2}+\alpha_{n} M_{1}, \quad \forall j \in \mathbb{N} .
$$

In view of Lemma 2.1 and (3.2), we obtain

$$
\begin{aligned}
\left\|x_{n+1}-z\right\|^{2} & =\left\|\alpha_{n} u+\left(1-\alpha_{n}\right) y_{n}-z\right\|^{2} \\
& =\left\|\alpha_{n}(u-z)+\left(1-\alpha_{n}\right)\left(y_{n}-z\right)\right\|^{2} \\
& \leq\left\|\left(1-\alpha_{n}\right)\left(y_{n}-z\right)\right\|^{2}+2\left\langle\alpha_{n}(u-z), J\left(x_{n+1}-z\right)\right\rangle \\
& =\left(1-\alpha_{n}\right)^{2}\left\|y_{n}-z\right\|^{2}+2\left\langle\alpha_{n}(u-z), J\left(x_{n+1}-z\right)\right\rangle \\
& \leq\left(1-\alpha_{n}\right)\left\|y_{n}-z\right\|^{2}+2\left\langle\alpha_{n}(u-z), J\left(x_{n+1}-z\right)\right\rangle \\
& =\left(1-\alpha_{n}\right)\left\|y_{n}-z\right\|^{2}+2 \alpha_{n}\left\langle u-z, J\left(x_{n+1}-z\right)\right\rangle .
\end{aligned}
$$

Step 3. We prove that $x_{n} \rightarrow z$ as $n \rightarrow \infty$.

We discuss the following two possible cases.

Case 1. Suppose that there exists $n_{0} \in \mathbb{N}$ such that $\left\{\left\|x_{n}-z\right\|\right\}_{n=n_{0}}^{\infty}$ is nonincreasing. Then the sequence $\left\{\left\|x_{n}-z\right\|\right\}_{n \in \mathbb{N}}$ is convergent. Thus we have $\left\|x_{n}-z\right\|^{2}-\left\|x_{n+1}-z\right\|^{2} \rightarrow 0$ as $n \rightarrow \infty$. This, together with condition (d) and (3.5), implies that

$$
\lim _{n \rightarrow \infty} g\left(\left\|x_{n}-T_{j} x_{n}\right\|\right)=0, \quad \forall j \in \mathbb{N} .
$$

From the properties of $g$, we conclude that

$$
\lim _{n \rightarrow \infty}\left\|x_{n}-T_{j} x_{n}\right\|=0, \quad \forall j \in \mathbb{N} .
$$


On the other hand, we have

$$
y_{n}-x_{n}=\sum_{j=1}^{\infty} \beta_{n, j}\left(T_{j} x_{n}-x_{n}\right) \text { and } x_{n+1}-y_{n}=\alpha_{n}\left(u-y_{n}\right) \text {. }
$$

This implies that

$$
\lim _{n \rightarrow \infty}\left\|y_{n}-x_{n}\right\|=0 \quad \text { and } \quad \lim _{n \rightarrow \infty}\left\|x_{n+1}-y_{n}\right\|=0
$$

By the triangle inequality, we conclude that

$$
\left\|x_{n+1}-x_{n}\right\| \leq\left\|x_{n+1}-y_{n}\right\|+\left\|y_{n}-x_{n}\right\|
$$

It follows from (3.7) that

$$
\lim _{n \rightarrow \infty}\left\|x_{n+1}-x_{n}\right\|=0
$$

Since $\left\{x_{n}\right\}_{n \in \mathbb{N}}$ is bounded, there exists a subsequence $\left\{x_{n_{i}}\right\}_{i \in \mathbb{N}}$ of $\left\{x_{n}\right\}_{n \in \mathbb{N}}$ such that $x_{n_{i}+1} \rightarrow$ $y \in C$ for all $j \in \mathbb{N}$. Since $T_{j}-I$ is demiclosed at 0 for each $j \in \mathbb{N}$, we conclude that $y \in F$. This, together with (2.1), implies that

$$
\begin{aligned}
\limsup _{n \rightarrow \infty}\left\langle u-z, J\left(x_{n+1}-z\right)\right\rangle & =\lim _{i \rightarrow \infty}\left\langle u-z, J\left(x_{n_{i}+1}-z\right)\right\rangle \\
& =\langle u-z, J(y-z)\rangle \\
& =\left\langle u-P_{F} u, J\left(y-P_{F} u\right)\right\rangle \\
& \leq 0 .
\end{aligned}
$$

Thus we have the desired result by Lemma 2.6 .

Case 2. Suppose that there exists a subsequence $\left\{n_{i}\right\}_{i \in \mathbb{N}}$ of $\{n\}_{n \in \mathbb{N}}$ such that

$$
\left\|x_{n_{i}}-z\right\|<\left\|x_{n_{i}+1}-z\right\|
$$

for all $i \in \mathbb{N}$. Then, by Lemma 2.7 , there exists a nondecreasing sequence $\left\{m_{k}\right\}_{k \in \mathbb{N}} \subset \mathbb{N}$ such that $m_{k} \rightarrow \infty$,

$$
\left\|z-x_{m_{k}}\right\|<\left\|z-x_{m_{k}+1}\right\| \text { and }\left\|z-x_{k}\right\| \leq\left\|x_{m_{k}+1}-\hat{u}\right\|
$$

for all $k \in \mathbb{N}$. This, together with (3.5), implies that

$$
\beta_{m_{k}, 0} \beta_{m_{k}, j} g\left(\left\|x_{m_{k}}-T_{j} x_{m_{k}}\right\|\right) \leq\left\|x_{m_{k}}-z\right\|^{2}-\left\|x_{m_{k}+1}-z\right\|^{2}+\alpha_{m_{k}} M_{1} \leq \alpha_{m_{k}} M_{1}
$$

for all $k \in \mathbb{N}$. Then, by conditions (a) and (d), we get

$$
\lim _{k \rightarrow \infty} g\left(\left\|x_{m_{k}}-T_{j} x_{m_{k}}\right\|\right)=0, \quad \forall j \in \mathbb{N} .
$$

From the properties of $g$, we conclude that

$$
\lim _{k \rightarrow \infty}\left\|x_{m_{k}}-T_{j} x_{m_{k}}\right\|=0, \quad \forall j \in \mathbb{N}
$$


By the same argument as Case 1, we arrive at

$$
\limsup _{k \rightarrow \infty}\left\langle u-z, J\left(x_{m_{k}}-z\right)\right\rangle=\limsup _{k \rightarrow \infty}\left\langle u-z, J\left(x_{m_{k}+1}-z\right)\right\rangle \leq 0 .
$$

It follows from (3.3) that

$$
\left\|x_{m_{k}+1}-z\right\|^{2} \leq\left(1-\alpha_{m_{k}}\right)\left\|x_{m_{k}}-z\right\|^{2}+\alpha_{m_{k}}\left\langle u-z, J\left(x_{m_{k}}-z\right)\right\rangle .
$$

Since $\left\|x_{m_{k}}-z\right\| \leq\left\|x_{m_{k}+1}-z\right\|$, we conclude that

$$
\begin{aligned}
\alpha_{m_{k}}\left\|x_{m_{k}}-z\right\|^{2} & \leq\left\|x_{m_{k}}-z\right\|^{2}-\left\|x_{m_{k}+1}-z\right\|^{2}+\alpha_{m_{k}}\left\langle u-z, J\left(x_{m_{k}}-z\right)\right\rangle \\
& \leq 2 \alpha_{m_{k}}\left\langle u-z, J\left(x_{m_{k}}-z\right)\right\rangle .
\end{aligned}
$$

In particular, since $\alpha_{m_{k}}>0$, we obtain

$$
\left\|x_{m_{k}}-z\right\|^{2} \leq\left\langle u-z, J\left(x_{m_{k}}-z\right)\right\rangle \text {. }
$$

In view of (3.11), we deduce that

$$
\lim _{k \rightarrow \infty}\left\|x_{m_{k}}-z\right\|=0
$$

This, together with (3.9), implies that

$$
\lim _{k \rightarrow \infty}\left\|x_{m_{k}+1}-z\right\|=0
$$

On the other hand, we have $\left\|x_{k}-z\right\| \leq\left\|x_{m_{k}+1}-z\right\|$ for all $k \in \mathbb{N}$, which implies that $x_{m_{k}} \rightarrow z$ as $k \rightarrow \infty$. Thus, we have $x_{n} \rightarrow z$ as $n \rightarrow \infty$, which completes the proof.

Considering a single mapping as a special case, we can obtain the following corollary directly, which extends and improves the main result of [17].

Corollary 3.1 Let E be a real uniformly convex Banach space which admits the weakly sequentially continuous duality mapping J. Let $C$ be a nonempty, closed and convex subset of $E$, let $T$ be a quasi-nonexpansive mapping from $C$ into itself, and let $F$ be the set of fixed points of $T$. Assume that $T-I$ is demiclosed at 0 . Let $\left\{\alpha_{n}\right\}_{n \in \mathbb{N}},\left\{\beta_{n}\right\}_{n \in \mathbb{N}, j \in \mathbb{N} \cup\{0\}}$ be sequences in $[0,1]$ satisfying the following control conditions:

(a) $\lim _{n \rightarrow \infty} \alpha_{n}=0$;

(b) $\sum_{n=1}^{\infty} \alpha_{n}=\infty$;

(c) $\liminf _{n \rightarrow \infty} \beta_{n}\left(1-\beta_{n}\right)>0$.

Let $\left\{x_{n}\right\}_{n \in \mathbb{N}}$ be a sequence generated by

$$
\left\{\begin{array}{l}
x_{1}, u \in C \quad \text { chosen arbitrarily, } \\
y_{n}=\beta_{n} x_{n}+\left(1-\beta_{n}\right) T x_{n} \\
x_{n+1}=\alpha_{n} u+\left(1-\alpha_{n}\right) y_{n} .
\end{array}\right.
$$

Then the sequence $\left\{x_{n}\right\}_{n \in \mathbb{N}}$ converges strongly to $P_{F} u$, where $P_{F}$ is the metric projection from E onto $F$. 
Remark 3.1 The main result of [17] gave a strong convergence theorem to approximate a fixed point of a nonexpansive mapping, while the main result of the present paper gives a strong convergence theorem to approximate common fixed points of an infinite family of quasi-nonexpansive mappings in a uniformly convex Banach space. We note that the proof of Theorem 3.1 (lines 2-5, where the authors used the nonexpansivity of the mapping $T$ ) in [17] is not valid in our discussion. We note also that the proof of Theorem 4 (where the author used the strong nonexpansivity of the mapping $T$ ) in [14] is not valid in our discussion. So our result extends and improves the corresponding results of [14, 17]. Corollary 3.1 also provides a positive answer to Open question 1.1 in a Banach space setting.

\section{Application (approximating zeroes of accretive operators)}

In this section, we propose a Halpern-type iterative scheme for finding common zeros of an infinite family of accretive operators in a uniformly convex Banach space and prove the following strong convergence theorem.

Theorem 4.1 Let E be a real uniformly convex Banach space which admits the weakly sequentially continuous duality mapping $J_{\phi}$. Let $\left\{A_{j}\right\}_{j=1}^{\infty}$ be an infinite family of accretive operators satisfying the range condition, and let $C$ be a nonempty, closed and convex subset of E such that $\overline{D\left(A_{j}\right)} \subset C \subset \bigcap_{r>0} R\left(I+r A_{j}\right)$ for each $j \in \mathbb{N}$. Let $r_{n}>0$ and $r>0$ be such that $\lim _{n \rightarrow \infty} r_{n}=r$, and let $\int_{r_{n}}^{A_{j}}=\left(I+r_{n} A_{j}\right)^{-1}$ be the resolvent of $A$. Let $\left\{\alpha_{n}\right\}_{n \in \mathbb{N}},\left\{\beta_{n, j}\right\}_{n \in \mathbb{N}, j \in \mathbb{N} \cup\{0\}}$ be sequences in $[0,1]$ satisfying the following control conditions:

(a) $\lim _{n \rightarrow \infty} \alpha_{n}=0$;

(b) $\sum_{n=1}^{\infty} \alpha_{n}=\infty$;

(c) $\beta_{n, 0}+\sum_{j=1}^{\infty} \beta_{n, j}=1, \forall n \in \mathbb{N}$;

(d) $\liminf _{n \rightarrow \infty} \beta_{n, 0} \beta_{n, j}>0, \forall j \in \mathbb{N}$.

Let $\left\{x_{n}\right\}_{n \in \mathbb{N}}$ be a sequence generated by

$$
\left\{\begin{array}{l}
x_{1}, u \in C \quad \text { chosen arbitrarily, } \\
y_{n}=\beta_{n, 0} x_{n}+\sum_{j=1}^{\infty} \beta_{n, j} j_{r_{n}}^{A_{j}} x_{n} \\
x_{n+1}=\alpha_{n} u+\left(1-\alpha_{n}\right) y_{n} .
\end{array}\right.
$$

If $Z:=\bigcap_{j=1}^{\infty} A_{j}^{-1}(0) \neq \emptyset$, then the sequence $\left\{x_{n}\right\}_{n \in \mathbb{N}}$ defined in (4.1) converges strongly to $Q_{Z} u$, where $Q_{Z}$ is the sunny nonexpansive retraction from $E$ onto $Z$.

Proof We divide the proof into several steps.

We first note that $Z$ is closed and convex. Set $z=Q_{Z} u$.

Step 1 . We prove that the sequences $\left\{x_{n}\right\}_{n \in \mathbb{N}},\left\{y_{n}\right\}_{n \in \mathbb{N}}$ and $\left\{\int_{r_{n}}^{A_{j}} x_{n}\right\}_{n \in \mathbb{N}}$ are bounded.

We first show that $\left\{x_{n}\right\}_{n \in \mathbb{N}}$ is bounded. Let $p \in Z$ be fixed. In view of Lemma 2.8, there exists a continuous, strictly increasing and convex function $g:[0, \infty) \rightarrow[0, \infty)$ with $g(0)=$ 0 such that for any $j \in \mathbb{N}$,

$$
\begin{aligned}
\left\|y_{n}-p\right\|^{2} & =\left\|\beta_{n, 0} x_{n}+\sum_{j=1}^{\infty} \beta_{n, j} j_{r_{n}}^{A_{j}} x_{n}-p\right\|^{2} \\
& \leq \beta_{n, 0}\left\|x_{n}-p\right\|^{2}+\sum_{j=1}^{\infty} \beta_{n, j}\left\|J_{r_{n}}^{A_{j}} x_{n}-p\right\|^{2}-\beta_{n, 0} \beta_{n, j} g\left(\left\|x_{n}-\int_{r_{n}}^{A_{j}} x_{n}\right\|\right)
\end{aligned}
$$




$$
\begin{aligned}
& \leq \beta_{n, 0}\left\|x_{n}-p\right\|^{2}+\sum_{j=1}^{\infty} \beta_{n, j}\left\|x_{n}-p\right\|^{2}-\beta_{n, 0} \beta_{n, j} g\left(\left\|x_{n}-\int_{r_{n}}^{A_{j}} x_{n}\right\|\right) \\
& =\left\|x_{n}-p\right\|^{2}-\beta_{n, 0} \beta_{n, j} g\left(\left\|x_{n}-\int_{r_{n}}^{A_{j}} x_{n}\right\|\right) \\
& \leq\left\|x_{n}-p\right\|^{2} .
\end{aligned}
$$

This implies that

$$
\begin{aligned}
\left\|x_{n+1}-p\right\| & =\left\|\alpha_{n} u+\left(1-\alpha_{n}\right) y_{n}-p\right\| \leq \alpha_{n}\|u-p\|+\left(1-\alpha_{n}\right)\left\|y_{n}-p\right\| \\
& \leq \alpha_{n}\|u-p\|+\left(1-\alpha_{n}\right)\left\|x_{n}-p\right\| \leq \max \left\{\|u-p\|,\left\|x_{n}-p\right\|\right\} .
\end{aligned}
$$

By induction, we obtain

$$
\left\|x_{n+1}-p\right\| \leq \max \left\{\|u-p\|,\left\|x_{1}-p\right\|\right\}
$$

for all $n \in \mathbb{N}$. This implies that the sequence $\left\{\left\|x_{n}-p\right\|\right\}_{n \in \mathbb{N}}$ is bounded and hence the sequence $\left\{x_{n}\right\}_{n \in \mathbb{N}}$ is bounded. This, together with (4.1), implies that the sequences $\left\{y_{n}\right\}_{n \in \mathbb{N}}$ and $\left\{r_{r_{n}}^{A_{j}} x_{n}\right\}_{n \in \mathbb{N}}$ are bounded too.

Step 2 . We prove that for any $n \in \mathbb{N}$,

$$
\left\|x_{n+1}-z\right\|^{2} \leq\left(1-\alpha_{n}\right)\left\|x_{n}-z\right\|^{2}+2 \alpha_{n}\left\langle u-z, J_{\phi}\left(x_{n+1}-z\right)\right\rangle .
$$

Let us show (4.3). For any $n, j \in \mathbb{N}$, in view of (4.2), we obtain

$$
\left\|y_{n}-z\right\|^{2} \leq\left\|x_{n}-z\right\|^{2}-\beta_{n, 0} \beta_{n, j} g\left(\left\|x_{n}-\int_{r_{n}}^{A_{j}} x_{n}\right\|\right) .
$$

This implies that

$$
\begin{aligned}
\left\|x_{n+1}-z\right\|^{2} & =\left\|\alpha_{n} u+\left(1-\alpha_{n}\right) y_{n}-z\right\|^{2} \leq \alpha_{n}\|u-z\|^{2}+\left(1-\alpha_{n}\right)\left\|y_{n}-z\right\|^{2} \\
& \leq \alpha_{n}\|u-z\|^{2}+\left(1-\alpha_{n}\right)\left[\left\|x_{n}-z\right\|^{2}-\beta_{n, 0} \beta_{n, j} g\left(\left\|x_{n}-\int_{r_{n}}^{A_{j}} x_{n}\right\|\right)\right] .
\end{aligned}
$$

Let $M_{2}:=\sup \left\{\left|\|u-z\|^{2}-\left\|x_{n}-z\right\|^{2}\right|+\beta_{n, 0} \beta_{n, j} g\left(\left\|x_{n}-\int_{r_{n}}^{A_{j}} x_{n}\right\|\right): n, j \in \mathbb{N}\right\}$. It follows from (4.4) that

$$
\beta_{n, 0} \beta_{n, j} g\left(\left\|x_{n}-J_{r_{n}}^{A_{j}} x_{n}\right\|\right) \leq\left\|x_{n}-z\right\|^{2}-\left\|x_{n+1}-z\right\|^{2}+\alpha_{n} M_{2}, \quad \forall j \in \mathbb{N} .
$$

In view of Lemma 2.1 and (4.2), we obtain

$$
\begin{aligned}
\left\|x_{n+1}-z\right\|^{2} & =\left\|\alpha_{n} u+\left(1-\alpha_{n}\right) y_{n}-z\right\|^{2} \\
& =\left\|\alpha_{n}(u-z)+\left(1-\alpha_{n}\right)\left(y_{n}-z\right)\right\|^{2} \\
& \leq\left\|\left(1-\alpha_{n}\right)\left(y_{n}-z\right)\right\|^{2}+2\left\langle\alpha_{n}(u-z), J_{\phi}\left(x_{n+1}-z\right)\right\rangle \\
& =\left(1-\alpha_{n}\right)^{2}\left\|y_{n}-z\right\|^{2}+2\left\langle\alpha_{n}(u-z), J_{\phi}\left(x_{n+1}-z\right)\right\rangle \\
& \leq\left(1-\alpha_{n}\right)\left\|y_{n}-z\right\|^{2}+2\left\langle\alpha_{n}(u-z), J_{\phi}\left(x_{n+1}-z\right)\right\rangle \\
& =\left(1-\alpha_{n}\right)\left\|y_{n}-z\right\|^{2}+2 \alpha_{n}\left\langle u-z, J_{\phi}\left(x_{n+1}-z\right)\right\rangle .
\end{aligned}
$$


Step 3. We prove that $x_{n} \rightarrow z$ as $n \rightarrow \infty$.

We discuss the following two possible cases.

Case 1. Suppose that there exists $n_{0} \in \mathbb{N}$ such that $\left\{\left\|x_{n}-z\right\|\right\}_{n=n_{0}}^{\infty}$ is nonincreasing. Then the sequence $\left\{\left\|x_{n}-z\right\|\right\}_{n \in \mathbb{N}}$ is convergent. Thus we have $\left\|x_{n}-z\right\|^{2}-\left\|x_{n+1}-z\right\|^{2} \rightarrow 0$ as $n \rightarrow \infty$. This, together with condition (d) and (4.5), implies that

$$
\lim _{n \rightarrow \infty} g\left(\left\|x_{n}-J_{r_{n}}^{A_{j}} x_{n}\right\|\right)=0, \quad \forall j \in \mathbb{N} .
$$

From the properties of $g$, we conclude that

$$
\lim _{n \rightarrow \infty}\left\|x_{n}-\int_{r_{n}}^{A_{j}} x_{n}\right\|=0, \quad \forall j \in \mathbb{N} .
$$

On the other hand, we have

$$
y_{n}-x_{n}=\sum_{j=1}^{\infty} \beta_{n, j}\left(J_{r_{n}}^{A_{j}} x_{n}-x_{n}\right) \quad \text { and } \quad x_{n+1}-y_{n}=\alpha_{n}\left(u-y_{n}\right) .
$$

This implies that

$$
\lim _{n \rightarrow \infty}\left\|y_{n}-x_{n}\right\|=0 \quad \text { and } \quad \lim _{n \rightarrow \infty}\left\|x_{n+1}-y_{n}\right\|=0 .
$$

By the triangle inequality, we conclude that

$$
\left\|x_{n+1}-x_{n}\right\| \leq\left\|x_{n+1}-y_{n}\right\|+\left\|y_{n}-x_{n}\right\| .
$$

It follows from (4.7) that

$$
\lim _{n \rightarrow \infty}\left\|x_{n+1}-x_{n}\right\|=0
$$

Exploiting Lemma 2.5 and (4.6), we obtain

$$
\left\|x_{n}-\int_{r}^{A_{j}} x_{n}\right\| \leq\left\|x_{n}-\int_{r_{n}}^{A_{j}} x_{n}\right\|+\left\|\int_{r_{n}}^{A_{j}} x_{n}-\int_{r}^{A_{j}} x_{n}\right\| \leq\left\|x_{n}-\int_{r_{n}}^{A_{j}} x_{n}\right\|+\frac{\left|r_{n}-r\right|}{r_{n}}\left\|x_{n}-\int_{r_{n}}^{A_{j}} x_{n}\right\| .
$$

This implies that

$$
\lim _{n \rightarrow \infty}\left\|x_{n}-J_{r}^{A_{j}} x_{n}\right\|=0, \quad \forall j \in \mathbb{N} .
$$

Since $\left\{x_{n}\right\}_{n \in \mathbb{N}}$ is bounded, there exists a subsequence $\left\{x_{n_{i}}\right\}_{i \in \mathbb{N}}$ of $\left\{x_{n}\right\}_{n \in \mathbb{N}}$ such that $x_{n_{i}+1} \rightarrow$ $y \in F\left(V_{r}^{A_{j}}\right)$ for all $j \in \mathbb{N}$. This, together with Lemma 2.1, implies that

$$
\begin{aligned}
\limsup _{n \rightarrow \infty}\left\langle u-z, J_{\phi}\left(x_{n+1}-z\right)\right\rangle & =\lim _{i \rightarrow \infty}\left\langle u-z, J_{\phi}\left(x_{n_{i}+1}-z\right)\right\rangle \\
& =\left\langle u-z, J_{\phi}(y-z)\right\rangle \\
& \leq 0 .
\end{aligned}
$$

Thus we have the desired result by Lemma 2.6 . 
Case 2. Suppose that there exists a subsequence $\left\{n_{i}\right\}_{i \in \mathbb{N}}$ of $\{n\}_{n \in \mathbb{N}}$ such that

$$
\left\|x_{n_{i}}-z\right\|<\left\|x_{n_{i}+1}-z\right\|
$$

for all $i \in \mathbb{N}$. Then, by Lemma 2.7, there exists a nondecreasing sequence $\left\{m_{k}\right\}_{k \in \mathbb{N}} \subset \mathbb{N}$ such that $m_{k} \rightarrow \infty$,

$$
\left\|z-x_{m_{k}}\right\|<\left\|z-x_{m_{k}+1}\right\| \quad \text { and } \quad\left\|z-x_{k}\right\| \leq\left\|x_{m_{k}+1}-\hat{u}\right\|
$$

for all $k \in \mathbb{N}$. This, together with (4.5), implies that

$$
\beta_{m_{k}, 0} \beta_{m_{k}, j} g\left(\left\|x_{m_{k}}-\int_{r}^{A_{j}} x_{m_{k}}\right\|\right) \leq\left\|x_{m_{k}}-z\right\|^{2}-\left\|x_{m_{k}+1}-z\right\|^{2}+\alpha_{m_{k}} M_{2} \leq \alpha_{m_{k}} M_{2}
$$

for all $k \in \mathbb{N}$. Then, by conditions (a) and (c), we get

$$
\lim _{k \rightarrow \infty} g\left(\left\|x_{m_{k}}-J_{r}^{A_{j}} x_{m_{k}}\right\|\right)=0, \quad \forall j \in \mathbb{N} .
$$

From the properties of $g$, we conclude that

$$
\lim _{k \rightarrow \infty}\left\|x_{m_{k}}-\int_{r}^{A_{j}} x_{m_{k}}\right\|=0, \quad \forall j \in \mathbb{N} .
$$

By the same argument as Case 1 , we arrive at

$$
\limsup _{k \rightarrow \infty}\left\langle u-z, J_{\phi}\left(x_{m_{k}}-z\right)\right\rangle=\limsup _{k \rightarrow \infty}\left\langle u-z, J_{\phi}\left(x_{m_{k}+1}-z\right)\right\rangle \leq 0 .
$$

It follows from (4.3) that

$$
\left\|x_{m_{k}+1}-z\right\|^{2} \leq\left(1-\alpha_{m_{k}}\right)\left\|x_{m_{k}}-z\right\|^{2}+\alpha_{m_{k}}\left\langle u-z, J_{\phi}\left(x_{m_{k}}-z\right)\right\rangle .
$$

Since $\left\|x_{m_{k}}-z\right\| \leq\left\|x_{m_{k}+1}-z\right\|$, we have that

$$
\begin{aligned}
\alpha_{m_{k}}\left\|x_{m_{k}}-z\right\|^{2} & \leq\left\|x_{m_{k}}-z\right\|^{2}-\left\|x_{m_{k}+1}-z\right\|^{2}+\alpha_{m_{k}}\left\langle u-z, J_{\phi}\left(x_{m_{k}}-z\right)\right\rangle \\
& \leq 2 \alpha_{m_{k}}\left\langle u-z, J_{\phi}\left(x_{m_{k}}-z\right)\right\rangle .
\end{aligned}
$$

In particular, since $\alpha_{m_{k}}>0$, we obtain

$$
\left\|x_{m_{k}}-z\right\|^{2} \leq\left\langle u-z, J_{\phi}\left(x_{m_{k}}-z\right)\right\rangle .
$$

In view of (4.12), we deduce that

$$
\lim _{k \rightarrow \infty}\left\|x_{m_{k}}-z\right\|=0 .
$$

This, together with (4.10), implies that

$$
\lim _{k \rightarrow \infty}\left\|x_{m_{k}+1}-z\right\|=0 .
$$


On the other hand, we have $\left\|x_{k}-z\right\| \leq\left\|x_{m_{k}+1}-z\right\|$ for all $k \in \mathbb{N}$, which implies that $x_{m_{k}} \rightarrow z$ as $k \rightarrow \infty$. Thus, we have $x_{n} \rightarrow z$ as $n \rightarrow \infty$, which completes the proof.

Theorem 4.2 Let $E$ be a real uniformly convex Banach space having a Gâteaux differentiable norm. Let $\left\{A_{j}\right\}_{j=1}^{\infty}$ be an infinite family of accretive operators satisfying the range condition, and let $C$ be a nonempty, closed and convex subset of $E$ such that $\overline{D\left(A_{j}\right)} \subset C \subset$ $\bigcap_{r>0} R\left(I+r A_{j}\right)$ for each $j \in \mathbb{N}$. Let $r_{n}>0$ and $r>0$ be such that $\lim _{n \rightarrow \infty} r_{n}=r$, and let $f_{r_{n}}^{A_{j}}=\left(I+r_{n} A_{j}\right)^{-1}$ be the resolvent of $A$. Let $\left\{\alpha_{n}\right\}_{n \in \mathbb{N}},\left\{\beta_{n, j}\right\}_{n \in \mathbb{N}, j \in \mathbb{N} \cup\{0\}}$ be sequences in $[0,1]$ satisfying the following control conditions:

(a) $\lim _{n \rightarrow \infty} \alpha_{n}=0$;

(b) $\sum_{n=1}^{\infty} \alpha_{n}=\infty$;

(c) $\beta_{n, 0}+\sum_{j=1}^{\infty} \beta_{n, j}=1, \forall n \in \mathbb{N}$;

(d) $\liminf _{n \rightarrow \infty} \beta_{n, 0} \beta_{n, j}>0, \forall j \in \mathbb{N}$.

Let $\left\{x_{n}\right\}_{n \in \mathbb{N}}$ be a sequence generated by

$$
\left\{\begin{array}{l}
x_{1} \in C \quad \text { chosen arbitrarily, } \\
y_{n}=\beta_{n, 0} x_{n}+\sum_{j=1}^{\infty} \beta_{n, j} J_{r_{n}}^{A_{j}} x_{n}, \\
x_{n+1}=\alpha_{n} u+\left(1-\alpha_{n}\right) y_{n} .
\end{array}\right.
$$

If $Z:=\bigcap_{j=1}^{\infty} A_{j}^{-1}(0) \neq \emptyset$, then the sequence $\left\{x_{n}\right\}_{n \in \mathbb{N}}$ converges strongly to $Q_{F} u$, where $Q_{F}$ is the sunny nonexpansive retraction from $E$ onto $Z$.

Remark 4.1 The main results of $[22,23]$ gave strong convergence theorems for finding common zeros of an accretive operator, while the main results of the present paper (Theorems 4.1 and 4.2) give strong convergence theorems for finding common zeros of an infinite family of accretive operators. So our results extend and improve the corresponding results of $[22,23]$.

Remark 4.2 The two-step Halpern iteration process is a generalization of the one-step Halpern iteration process. It provides more flexibility in defining the algorithm parameters, which is important from the numerical implementation perspective.

Competing interests

The authors declare that they have no competing interests.

Authors' contributions

All authors read and approved the final manuscript.

Author details

${ }^{1}$ Department of Information Management, Yuan Ze University, Chung-Li, 32003, Taiwan. ${ }^{2}$ Department of Mathematics, Yasouj University, Yasouj, 75918, Iran.

Received: 10 June 2013 Accepted: 18 November 2013 Published: 05 Dec 2013

References

1. Takahashi, W: Nonlinear Functional Analysis, Fixed Point Theory and Its Applications. Yokahama Publishers, Yokahama (2000)

2. Gossez, JP, Dozo, EL: Some geometric properties related to the fixed point theory for nonexpansive mappings. Pac. J. Math. 40, 565-573 (1972)

3. Reich, S: Geometry of Banach spaces, duality mappings and nonlinear problems. Bull. Am. Math. Soc. 26, 367-370 (1992)

4. Halpern, B: Fixed points of nonexpanding mappings. Bull. Am. Math. Soc. 73, 957-961 (1967) 
5. Reich, S: Approximating fixed points of nonexpansive mappings. Panam. Math. J. 4, 23-28 (1994)

6. Reich, S: Strong convergence theorems for resolvents of accretive operators in Banach spaces. J. Math. Anal. Appl. 75, 287-292 (1980)

7. Lions, PL: Approximation de points fixes de contractions. C. R. Acad. Sci. Paris, Ser. A-B 284, A1357-A1359 (1977)

8. Wittmann, R: Approximation of fixed points of nonexpansive mappings. Arch. Math. 58, 486-491 (1992)

9. Shoiji, N, Takahashi, W: Strong convergence of approximated sequences for nonexpansive mappings in Banach spaces. Proc. Am. Math. Soc. 125, 3641-3645 (1997)

10. Xu, H-K: Another control condition in an iterative method for nonexpansive mappings. Bull. Aust. Math. Soc. 65 109-113 (2002)

11. Cho, YJ, Kang, SM, Zhou, H: Some control conditions on iterative methods. Commun. Appl. Nonlinear Anal. 12, 27-34 (2005)

12. Suzuki, T: A sufficient and necessary condition for Halpern-type strong convergence to fixed points of nonexpansive mappings. Proc. Am. Math. Soc. 135, 99-106 (2007)

13. Nilsrakoo, W, Saejung, S: Strong convergence theorems by Halpern-Mann iterations for relatively nonexpansive mappings in Banach spaces. Appl. Math. Comput. 217, 6577-6586 (2011)

14. Saejung, S: Halpern's iteration in Banach spaces. Nonlinear Anal. 73, 3431-3439 (2010)

15. Song, $Y$, Chai, $X$ : Halpern iteration for firmly type nonexpansive mappings. Nonlinear Anal. 71, 4500-4506 (2009)

16. Suzuki, T: Reich's problem concerning Halpern's convergence. Arch. Math. 92, 602-613 (2009)

17. Yao, Y, Liou, Y-C, Zhou, H: Strong convergence of an iterative method for nonexpansive mappings with new control conditions. Nonlinear Anal. 70, 2332-2336 (2009)

18. Bruck, RE, Reich, S: Nonexpansive projections and resolvents of accretive operators in Banach spaces. Houst. J. Math 3, 459-470 (1997)

19. Nevanlinna, O, Reich, S: Strong convergence of contraction semigroups and of iterative methods for accretive operators in Banach spaces. Isr. J. Math. 32, 44-58 (1997)

20. Lopez, G, Martin-Marquez, V, Xu, H-K: Halpern's iteration for nonexpansive mappings. Contemp. Math. 513, 211-231 (2010)

21. $\mathrm{Xu}, \mathrm{H}-\mathrm{K}$ : Strong convergence of an iterative method for nonexpansive and accretive operators. J. Math. Anal. Appl. 314, 631-643 (2006)

22. Zhang, Q, Song, Y: Halpern type proximal point algorithm of accretive operators. Nonlinear Anal. 75, 1859-1868 (2012)

23. Yu, Y: Convergence analysis of a Halpern type algorithm for accretive operators. Nonlinear Anal. 75, 5027-5031 (2012)

24. Goebel, K, Reich, S: Uniform Convexity, Hyperbolic Geometry, and Nonexpansive Mappings. Dekker, New York (1984)

25. Reich, S: Asymptotic behavior of contractions in Banach spaces. J. Math. Anal. Appl. 44, 57-70 (1973)

26. Browder, FE: Convergence theorems for sequences of nonlinear operators in Banach spaces. Math. Z. 100, 201-225 (1967)

27. Reich, S: Approximating zeros of accretive operators. Proc. Am. Math. Soc. 51, 381-384 (1975)

28. Goebel, K, Kirk, WA: Topics in Metric Fixed Point Theory. Cambridge Studies in Advanced Mathematics, vol. 28 Cambridge University Press, Cambridge (1990)

29. Xu, H-K, Kim, TK: Convergence of hybrid steepest-descent methods for variational inequalities. J. Optim. Theory Appl. 119(1), 185-201 (2003)

30. Maingé, PE: Strong convergence of projected subgradient methods for nonsmooth and nonstrictly convex minimization. Set-Valued Anal. 16, 899-912 (2008)

31. Chang, SS, Kim, JK, Wang, XR: Modified block iterative algorithm for solving convex feasibility problems in Banach spaces. J. Inequal. Appl. 2010, Article ID 869684 (2010)

10.1186/1687-1812-2013-332

Cite this article as: Pang and Naraghirad: Strong convergence of Halpern iterations for quasi-nonexpansive mappings and accretive operators in Banach spaces. Fixed Point Theory and Applications 2013, 2013:332

\section{Submit your manuscript to a SpringerOpen ${ }^{\circ}$ journal and benefit from:}

- Convenient online submission

Rigorous peer review

- Immediate publication on acceptance

- Open access: articles freely available online

- High visibility within the field

- Retaining the copyright to your article 